\title{
Sistema para Aquisição e Análise de Dados de Velocidade e Direção do Ar Utilizando Scilab e Arduino
}

\author{
Marcus Vinicius Ferreira da Silva \\ Centro de Ciências Exatas e Tecnológicas \\ Universidade Federal do Acre - UFAC \\ Rio Branco, Brasil \\ e-mail: vfsmarcus@gmail.com
}

\author{
Tiago Franscisconi Borges Camargo \\ Centro de Ciências Exatas e Tecnológicas \\ Universidade Federal do Acre - UFAC \\ Rio Branco, Brasil \\ e-mail: tiagofbcamargo@gmail.com
}

\begin{abstract}
Resumo-Este projeto aborda o desenvolvimento de um sistema de aquisição de dados com foco na medição e análise de velocidade e direção do vento, utilizando modelamento em ambiente computacional de softwares livres, componentes de baixo custo e a plataforma de prototipagem Arduino para o desenvolvimento do protótipo final. O software livre utilizado para a análise de dados deste projeto foi o Scilab, software científico para computação numérica semelhante ao MATLAB, que fornece um poderoso ambiente computacional aberto para aplicações científicas. O Scilab foi então conectado à placa Arduino através de comunicação serial, visando a troca de dados adquiridos por meio de sensores implementados no protótipo físico final.
\end{abstract}

Palavras-chave: Scilab; Arduino; Aquisição de dados; Anemômetro.

\begin{abstract}
This project addresses the development of a data acquisition system focused on measurement and analysis of speed and direction of wind, using computational modeling of free software, low cost components and a prototyping platform Arduino. The free software used for this project's data analysis was Scilab, scientific software for numeric computation similar to MATLAB, which provides a powerful open computing environment for scientific applications. The Scilab was then connected to the Arduino board through serial communication, aiming at an exchange of data which were acquired through sensors implemented in the final physical prototype.
\end{abstract}

Keywords: Scilab; Arduino; Data acquisition; Anemometer.

\section{INTRODUÇÃO}

$\mathrm{O}$ vento consiste no deslocamento de massas de ar, sendo que esse fenômeno é consequência do movimento do ar de um ponto no qual a pressão atmosférica é mais alta em direção a um ponto onde ela é mais baixa. Os principais elementos que interferem na pressão atmosférica são a temperatura e a altitude: zonas de baixa altitude = zona de alta pressão atmosférica; zona de elevada altitude = zona de baixa pressão atmosférica. Os ventos são de fundamental importância na dinâmica terrestre, visto que eles são modeladores do relevo, transportam umidade dos oceanos para as porções continentais, amenizam o calor das zonas de baixa pressão atmosférica, entre outros fatores.

Além da enorme importância para a natureza e para a dinâmica terrestre, nos dias atuais o vento é muito importante também para a humanidade, sendo utilizado para os mais diversos fins, como navegação e geração de energia elétrica, por exemplo. Logo, conhecer sua intensidade e também a direção pela qual flui é de grande utilidade, sendo determinante na geração eólica para calcular com precisão a quantidade de energia elétrica que está sendo gerada pelas enormes pás de um aerogerador, e na navegação para se obter a noção de qual a melhor direção a ser seguida. Outra aplicação deste projeto, além das duas citadas anteriormente, é na agricultura, pois os ventos excessivos são um grande problema nas áreas rurais, já que influenciem diretamente no crescimento das culturas. Sendo assim, se faz necessário o monitoramento da velocidade e da direção do vento por parte do produtor.

$\mathrm{O}$ anemômetro é um dispositivo meteorológico usado para medir a velocidade e intensidade dos ventos, ou de outros fluidos em movimento, e existem diversos tipos e modelos no mercado, como os anemômetros de copo, termoelétricos e ultrassônicos. A ideia deste projeto é desenvolver um tipo de medidor que seja uma alternativa aos anemômetros já existentes no mercado, fazendo uso de sensores infravermelho e ferramentas computacionais como Scilab e Arduino, objetivando um projeto consideravelmente mais barato e que atinja resultados semelhantes ao de equipamentos mais sofisticados, estes que acabam possuindo preço bem elevado.

A engenharia busca utilizar a matemática e a física em sua forma aplicada, tornando extremamente útil a utilização de ferramentas computacionais para automatizar o desenvolvimento de soluções para os mais diversos problemas, como o proposto para este projeto, por exemplo. O uso de softwares pagos, como o MATLAB, por exemplo, agrega enorme valor tecnológico, sendo extremamente importante para avanços na humanidade, porém, muitos softwares pagos podem substituídos por softwares livres, ou seja, softwares que estão disponíveis sem nenhum custo, como o Scilab, e que podem apresentar os mesmo avanços para obtenção de dados no uso comercial e científico. Os softwares livres são ferramentas que auxiliam tanto estudantes universitários como profissionais especializados, devido à sua abrangência de conteúdos relevantes, sua facilidade de divulgação e custo em licenças. Neste projeto, utilizamos o Scilab para realizar a análise de dados, exibindo por meio de gráficos em tempo real os valores atuais de velocidade e direção do ar.

Outra parte extremamente importante para o desenvolvimento deste projeto foi a utilização da placa de prototipagem Arduino, uma ferramenta projetada com a intenção de permitir que um computador pessoal interaja com o mundo físico. É uma plataforma física de códigofonte aberto, baseada em um microcontrolador adicionado a um ambiente de desenvolvimento de software livre para instalá-los na placa. Este dispositivo permite o desen- 
volvimento de objetos interativos, entradas de vários sensores e luzes de controle, motores e outros dispositivos e pode ser usado sozinho ou em comunicação com outros softwares instalados no computador. Neste projeto utilizamos o Arduino para converter os dados analógicos, obtidos através de sensores infravermelho implementados no protótipo físico, em dados digitais a serem analisados.

\section{OBJETIVO GERAL}

Desenvolvimento de um sistema de aquisição de dados com foco na medição e análise de velocidade e direção do vento, utilizando modelamento em ambiente computacional no software livre de computação numérica Scilab e na plataforma de prototipagem Arduino, além de componentes de baixo custo para o desenvolvimento do protótipo final.

\section{OBJETIVOS ESPECÍFICOS}

- Realizar revisão dos softwares livres ou plataformas existentes e sua aplicação;

- Expor um resumo dos comandos de programação comuns de softwares eleitos a serem utilizados nos cálculos e possíveis simulações;

- Definir equipamentos que podem ser comparados ou que são usados na região de Rio Branco em estações climáticas;

- Fundamentar os métodos com equações usuais para cada tipo de captação;

- Obter dados através de plataforma de código aberto juntamente com sensores;

- Aplicar nos softwares livres as equações e possíveis recursos existentes para automatizar cálculos;

- Armazenar e tratar dados;

- Utilizar dados obtidos em bibliografia para comparar com resultados obtidos.

\section{MetOdOLOGIA}

Inicialmente foi realizada uma pesquisa e revisão bibliográfica para o projeto, com o objetivo de se construir uma base sólida para a sua execução. Para isso foram utilizados livros com foco na área de aplicação deste projeto e também artigos científicos.

Vencida esta etapa, iniciou-se o desenvolvimento dos códigos em Arduino e também Scilab, bem como a compra dos materiais necessários para os primeiros testes, como resistores, sensores, jumpers e protoboard, além da utilização de uma placa de prototipagem Arduino, já pertencente ao autor do projeto. Como o nome no projeto deixa claro, foram utilizados materiais de baixo custo, porém, com ótima funcionalidade, para cumprir de forma eficiente o objetivo do projeto. Também foram adquiridos materiais a custo zero, por meio de reciclagem, como isopor, bolinha de desodorante e fita isolante.

Perto do fim da primeira metade do projeto, foi então implementado um protótipo para a realização de testes, utilizando os códigos já desenvolvidos e aprimorados desde o início, com o objetivo de se observar o que já havia sido feito até então. $\mathrm{O}$ resultado foi promissor, cumprindo com o esperado para o primeiro teste, porém, ainda bastante distante do protótipo final.

Para realizarmos a medição de velocidade com precisão, utilizamos sensores QRD1114, mostrado na Fig. 1, que é um sensor composto por um LED infravermelho e um fototransistor infravermelho para detecção de um sinal infravermelho refletido, ideal para utilização em projetos de seguidores de linha e para detecção de obstáculos que estejam a uma distância de 0.5 a $1 \mathrm{~cm}$.

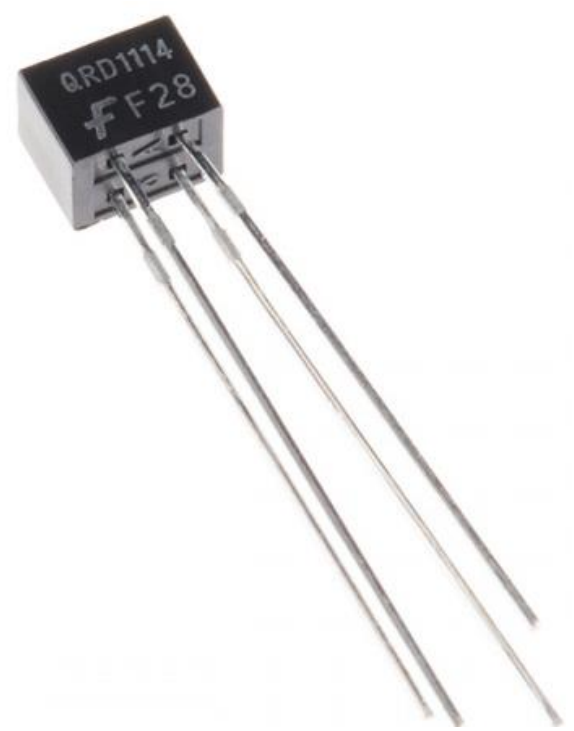

Figura 1: Sensor QRD1114

O protótipo físico construído para o projeto é composto basicamente de uma carcaça oca de ferro com formato de paralelepípedo, onde em seu interior existem dois cubos de isopor: um conectado a uma bolinha de desodorante, com a função de servir como obstáculo para dois sensores QRD1114 instalados perpendicularmente nas laterais do protótipo, servindo assim para medir deslocamentos nos eixos $\mathrm{x}$ e $\mathrm{y}$, e outro cubo servindo para sustentar $\mathrm{o}$ primeiro na posição central por meio de linhas de costura comum, desta forma será possível medir a velocidade do vento de acordo com variações de posição do primeiro cubo em relação aos sensores por conta da incidência de vento na bolinha, ou seja, fazendo o equacionamento e encontrando uma função que descreva matematicamente a relação entre a distância do cubo para os sensores e a velocidade do vento no local onde o protótipo está instalado.

Os dados de distância de cada sensor foram obtidos através de um Arduino Uno, conectando cada sensor de acordo com a Fig. 2, alterando apenas a porta analógica utilizado, que será A0 para o primeiro sensor e A1 para o segundo. 


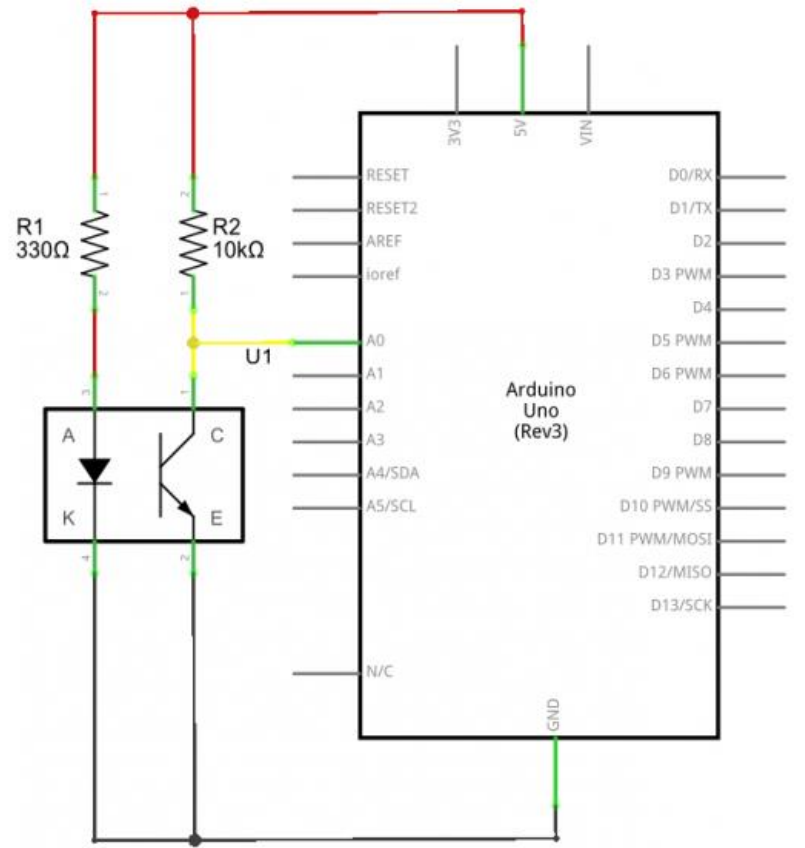

Figura 2: Diagrama de conexão para QRD1114 e Arduino Uno

Para obter-se uma melhor precisão nos valores recebidos pelos sensores, foi utilizado um Filtro de Média Móvel. Dada uma série de valores e um subconjunto finito destes, o primeiro elemento de uma média móvel é a média deste subconjunto. $\mathrm{O}$ próximo elemento é a média de todos os elementos do subconjunto menos o primeiro e mais o primeiro elemento do próximo subconjunto "à direita" na série. Médias móveis são comumente usadas com séries temporais para suavizar flutuações curtas e destacar tendências de longo prazo, dessa forma teremos menos "ruído" nos dados dos sensores. Após a filtragem, os dados são então enviados ao Scilab por meio de comunicação serial.

Porém, o dado recebido de cada sensor pelo Arduino é um valor em bits, logo, precisamos converter esse valor para centímetros. A conversão é feita apenas no Scilab, primeiro transformando o valor para tensão, apenas multiplicando o valor recebido por 5 e em seguida dividindo o resultado por 1023, que é a quantidade total de bits. Em seguida, para finalmente termos o valor em centímetros, dividimos o valor encontrado em tensão por 4.8, que é o valor da tensão limite do sensor, ou seja, quando ele está indicando uma distância de $1 \mathrm{~cm}$.

Porém, como o sensor mede distâncias de 0 a $1 \mathrm{~cm}$, e precisamos de uma escala em que o cubo ateste uma distância de $0 \mathrm{~cm}$ quando estiver centralizado no protótipo, ou seja, quando não houver vento incidindo. Dessa forma, para cada valor de distância calculado foi subtraído o valor de $0.5 \mathrm{~cm}$, ou seja, o sensor atestará agora distâncias que vão de $-0.5 \mathrm{~cm}$ à $0.5 \mathrm{~cm}$. Logo, com $0 \mathrm{~cm}$ também teremos $0 \mathrm{~m} / \mathrm{s}$ de velocidade.

Após a conversão para centímetros, foi feito o o ajuste de curva que relaciona a distância e velocidade do vento. Para isso foi utilizado um multímetro profissional, regulado para a função de anemômetro, e um ventilador comum, tomando medições na parte traseira do mesmo, onde temos escoamento uniforme e sem turbulências devido às pás, aumentando a precisão do sistema. Para cada valor de velocidade (em $\mathrm{m} / \mathrm{s})$, atestado com o anemômetro, foi constatado um deslocamento do cubo no interior da carcaça em relação aos sensores. Este processo foi realizado para ventos incidindo nas direções positiva e negativa de cada sensor, ou seja, obtivemos quatro equações, que foram encontradas após aplicar os dados no editor de planilhas Excel e plotar-se os gráficos, alcançando mais $99 \%$ de precisão em todas as equações.

Com os valores de velocidade de cada eixo em mãos, o próximo passo foi encontrar o seu valor em módulo, para que assim pudéssemos obter o valor real da velocidade independente da direção de incidência do vento. Como temos componentes de velocidade em x e y, calculando-se a raiz quadrada da soma de cada componente ao quadrado obtêm-se a velocidade total em módulo. Além da intensidade da velocidade do vento, também precisamos determinar a sua direção. Para isso realizamos o cálculo do arco tangente da componente em y dividida pela componente em x para a distância, sendo o ângulo em graus o resultado da operação. De acordo com o valor em graus, o código informa qual a direção de incidência do vento. Finalizando o processo, é realizada a plotagem dos dados calculados em tempo real no Scilab.

\section{Resultados E Discuss Ão}

Após a construção do protótipo inicial para a realização de testes na primeira metade do projeto, foram feitas algumas alterações no mesmo para finalmente chegarmos ao protótipo final, visto na Fig. 3, que possui todas as especificações necessárias e funciona de acordo com o exigido para o projeto.

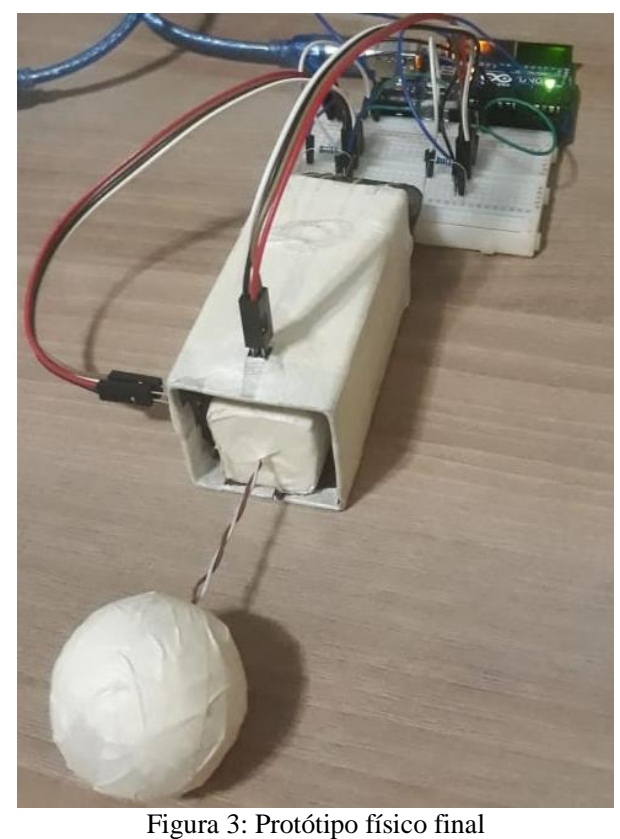

Na Fig. 4 podemos observar o filtro de média móvel operando para um dos sensores QRD1114 a medida que cubo se aproxima e se afasta do sensor. Foi utilizado o Plotter Serial da IDE do Arduino para a visualização. 
O sinal em azul é o sinal antes da filtragem, e o sinal em vermelho é o sinal suavizado após a filtragem.

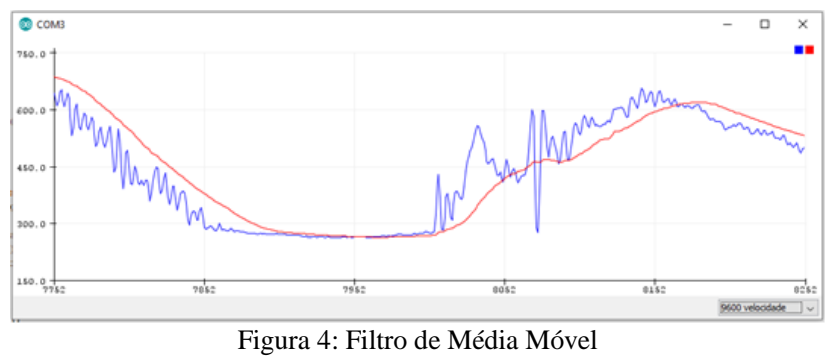

Como dito anteriormente, após a filtragem dos dados na própria IDE do Arduino, os dados foram transferidos para o Scilab, onde foi realizada a conversão para centímetros. Somente após esta etapa foram realizados os testes com o ventilador e anemômetro para efetuar o ajuste de curva, interpolando os dados obtidos. Os dados, gráficos e equações estão mostradas nas Fig. 5 e 6.

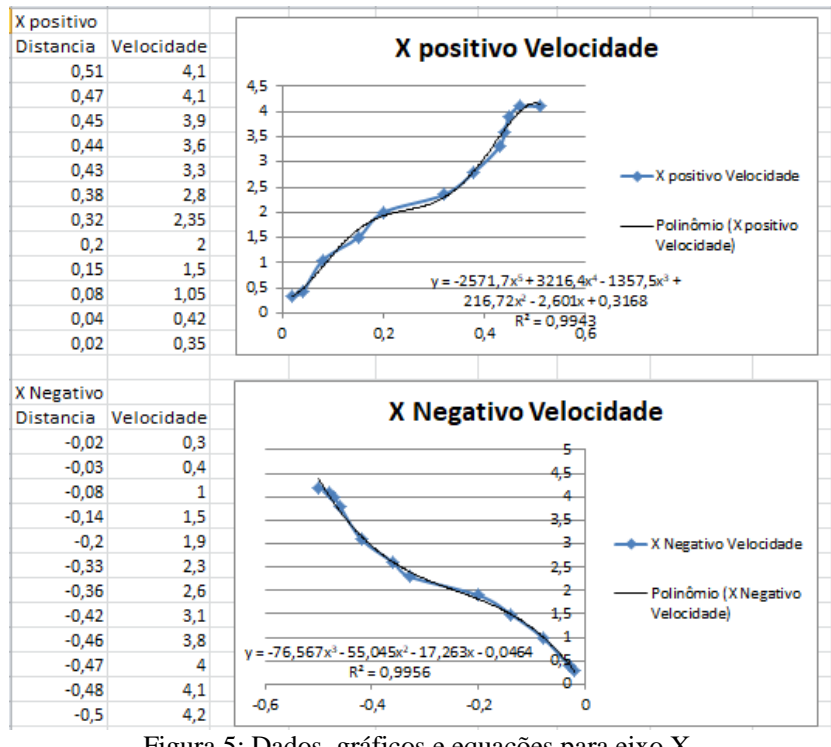

Figura 5: Dados, gráficos e equações para eixo $\mathrm{X}$

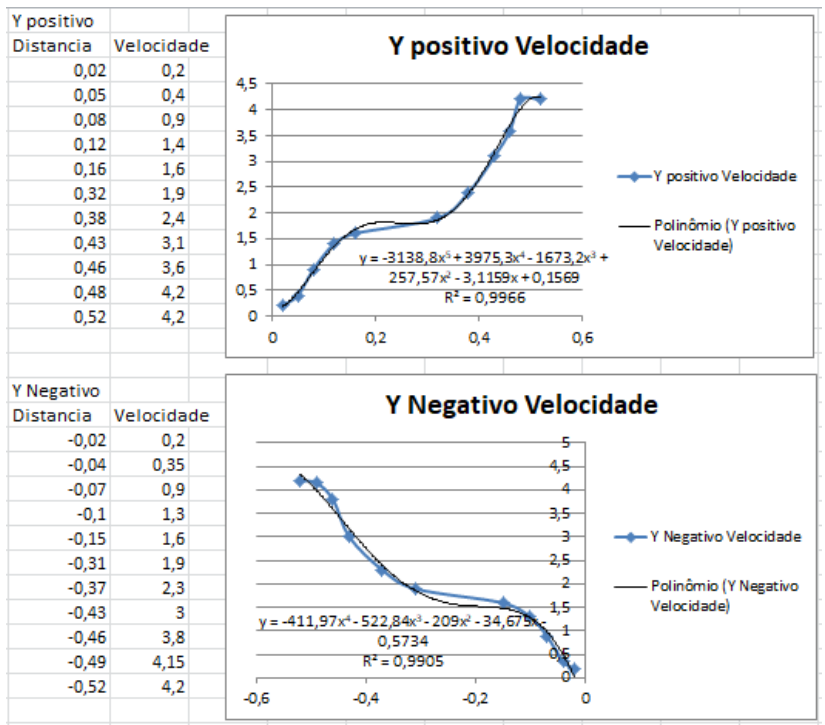

Figura 6: Dados, gráficos e equações para eixo $\mathrm{Y}$
Com todos os dados necessários em mãos, o próximo passo foi a finalização dos códigos em Arduino e Scilab. Após isso, foram realizados testes utilizando o protótipo finalizado em frente ao ventilador.

Para um valor de $4.6 \mathrm{~m} / \mathrm{s}$ de fluxo de ar, medido com um anemômetro profissional em frente a um ventilador comum na velocidade "3", na direção Norte do sensor, encontrou-se amostras com maior constância de valores entre $4.3 \mathrm{~m} / \mathrm{s}$ e $4.55 \mathrm{~m} / \mathrm{s}$ para o módulo de velocidade, com o protótipo indicando uma angulação entre $52.9^{\circ}$ e $90.7^{\circ}$, ou seja, indicando a direção Norte, de acordo com o previsto, apesar de haver erros levemente maiores que $5 \%$ entre o valor medido neste projeto e o valor atestado pelo anemômetro profissional. As amostras obtidas podem ser observadas na Fig. 7.

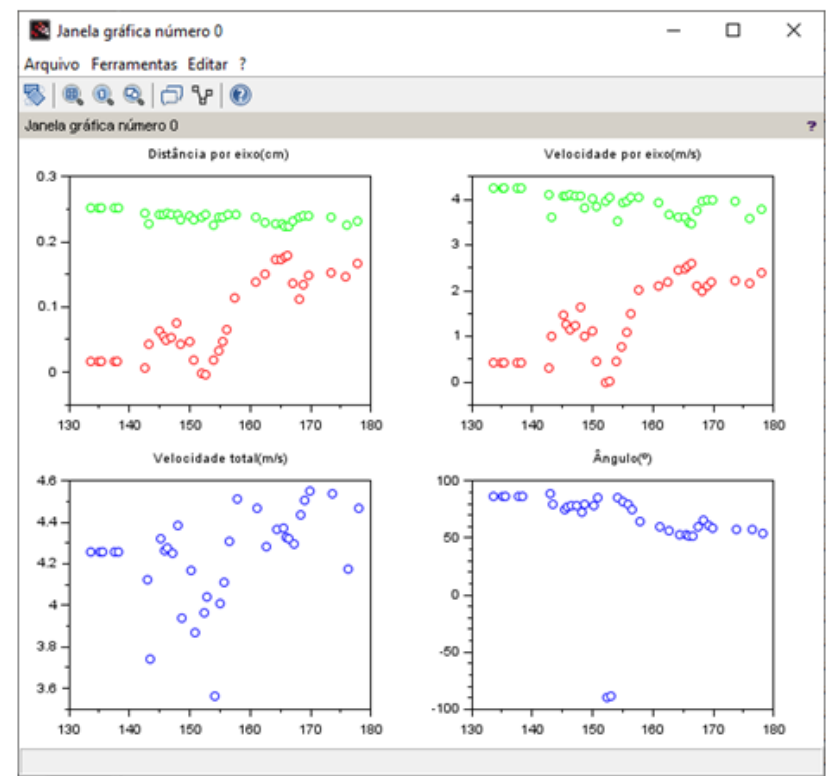

Figura 7: Resultados obtidos para velocidade de $4.6 \mathrm{~m} / \mathrm{s}$ na direção Norte

Um segundo teste foi realizado para testar a real funcionalidade do projeto, desta vez para um fluxo na direção Nordeste com valor de $5.6 \mathrm{~m} / \mathrm{s}$, medido em frente ao mesmo ventilador na velocidade "4". Os resultados foram de maior constância para valores entre $5.3 \mathrm{~m} / \mathrm{s}$ e 5.8 $\mathrm{m} / \mathrm{s}$, com o valor real estando inserido neste intervalo. Para a angulação, obtivemos valores entre $36.3^{\circ}$ e $44.8^{\circ}$, indicando perfeitamente a direção Nordeste. As amostras obtidas estão expostas na Fig. 8. 


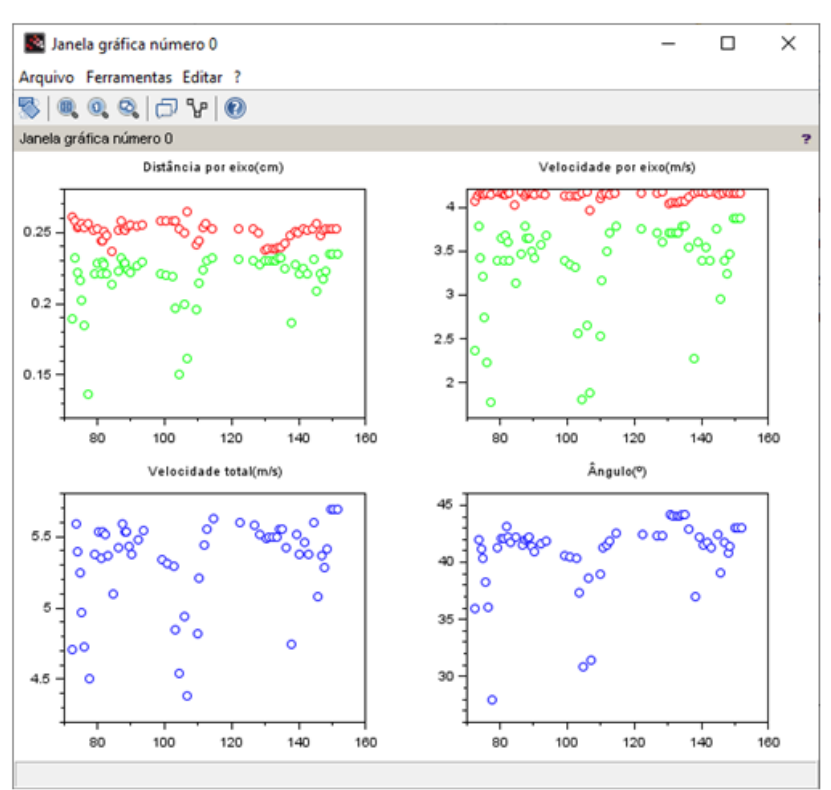

Figura 8: Resultados obtidos para velocidade de $5.6 \mathrm{~m} / \mathrm{s}$ na direção Nordeste

\section{CONCLUSÃO}

O protótipo final obteve resultados bem próximos aos encontrados com anemômetros disponíveis no mercado por preços bem mais elevados do que gasto para a implementação deste projeto, sendo capaz de medir com precisão velocidades de 0 à $5.9 \mathrm{~m} / \mathrm{s}$ em oito direções, como Norte e Noroeste e podendo ser utilizado para aplicações nas áreas de geração de energia elétrica em usinas eólicas e navegação, ainda que o mesmo apresente pequenos erros percentuais de até $5 \%$ em relação ao valor medido com outros aparelhos.

Além disso, através deste projeto pôde-se observar a importância da utilização de ferramentas computacionais para resolução de problemas na área de engenharia. Os softwares livres, como o Scilab utilizado neste projeto, ajudam na diminuição do trabalho manual que poderia ser exigido para a solução de um problema e aumentam a velocidade em que se chega aos resultados, além de facilitar a visualização de tais resultados por meio de gráficos em tempo real. Dessa forma, o que foi aprendido sobre a utilização de ferramentas como Arduino e Scilab durante a execução deste projeto poderá ser utilizado em projetos futuros como medições de temperatura e pressão, por exemplo, com armazenamento dos dados e apresentação em tempo real na internet.

\section{AGRADECIMENTOS}

Agradecemos à Universidade Federal do Acre (UFAC), pela oportunidade e disponibilização de recursos através de um projeto de iniciação científica (PIBIC) para a realização deste artigo.

\section{REFERÊNCIAS}

[1] SILVEIRA, Luciano Roberto da. "Sistema de aquisição de dados para equipamentos de medição da permeabilidade ao ar do solo". Rev. Bras. Ciênc. Solo, Viçosa, v.35, n.2, p.429-436, Abril de 2011. Disponível em: http://www.scielo.br/scielo.php?script=sci arttext\&pid=S010006832011000200012\&lng=en\&nrm=iso Acesso em 28 de Agosto de 2018, in press.

[2] SILVA, Wellington Leite da; CHOQUE, Nilo M.S. "Desenvolvimento de sistema de aquisição de dados usando um software placa Arduino Uno e NI-LABVIEW". Disponível em: https://doaj.org/article/333fafdfda3241fba5fa3bf9071fba80. Acesso em 30 de Agosto de 2018, in press.

[3] BONILLA, Maria Helena Silveira. "Software Livre e Educação: uma relação em construção". Disponível em: https://doaj.org/article/2cbbebb1969d4fe8aed28e03110054 d8 Acesso em 31 de Agosto de 2018, in press

[4] MORAES, Jermanda Lopes. "Sistema de aquisição de dados para a máquina de impacto Charpy". Disponível em: https://doaj.org/article/bf5db740a8ef430484d4 0322b7d2ab37. Acesso em 2 de Setembro de 2018, in press.

[5] FRANCISCO, Wagner de Cerqueria e. "Vento"; Brasil Escola Disponível em: https://brasilescola.uol.com.br/geografia/vento.htm. Acesso em 15 de Junho de 2019, in press.

[6] O Sistema Operacional GNU. "O que é o software livre?". Disponível em: https://www.gnu.org/philosophy/free-sw.ptbr.html. Acesso em 4 de Setembro de 2018.

[7] Instrusul. "Para que serve o anemômetro". Disponível em: http://blog.instrusul.com.br/para-que-serve-o-anemometro/. Acesso em 15 de Junho de 2019.

[8] National Instruments. “O que é aquisição de dados?”. Disponível em: http://www.ni.com/data-acquisition/what-is/pt/. Acesso em 28 de Agosto de 2018.

[9] Sparkfun. "QRD1114 Optical Detector Hookup Guide". Disponível em: https://learn.sparkfun.com/tutorials/qrd1114 optical-detector-hookup-guide/all. Acesso em 28 de Novembro de 2018.

[10] Leandroip. "Scilab e Arduino - O básico". Disponível em: http://leandroip.com/index.php/2018/06/15/scilab-e-arduino-obasico/. Acesso em 02 de Dezembro de 2018 